\title{
Diffuse Alveolar Infiltrates and Macroscopic Hematuria: A Run-and-Gun Affair
}

\author{
Lykurgos Kolilekas $^{a} \quad$ Christina Triantafillidou $^{a}$ Ioannis Vrettos ${ }^{b}$ \\ Velissarios Gkikas $^{b}$ Ioannis Kalomenidis ${ }^{a}$ Effrosyni Manali ${ }^{a}$ Lydia Nakopoulou ${ }^{c}$ \\ Spyros Papiris ${ }^{a}$
}

a 2nd Pulmonary Department and b 2nd Department of Internal Medicine, 'Attikon' University Hospital, and

'1st Department of Pathology, Athens Medical School, National and Kapodistrian University of Athens,

Athens, Greece

\section{Case Report}

A 40-year-old taxi driver, current smoker and previously in good health, was admitted because of fever, cough with blood-tinged sputum and dyspnea of 5 days' duration. Two weeks prior to admission the patient developed a sore throat and tonsillar swelling. On admission, the patient was febrile $\left(38.5^{\circ} \mathrm{C}\right)$, with dyspnea on exertion (category 3 of the modified Medical Research Council Dyspnea Scale) and the blood pressure was 140/95 mm $\mathrm{Hg}$. Arterial blood gas analysis showed $\mathrm{pH} 7.39, \mathrm{Po}_{2} 7.8$ $\mathrm{kPa}(59 \mathrm{~mm} \mathrm{Hg}), \mathrm{PCO}_{2} 4.6 \mathrm{kPa}(35 \mathrm{~mm} \mathrm{Hg}), \mathrm{HCO}_{3}^{-} 22$ $\mathrm{mEq} / \mathrm{l}$ and $\mathrm{S}_{\mathrm{a}} \mathrm{O}_{2} 90 \%$ on room air. Chest auscultation revealed inspiratory crackles at both mid and lower lung fields. The remainder of the physical examination was unremarkable, except slight lower limbs edema and 1-2 $\mathrm{kg}$ gain of body weight as referred by the patient. Laboratory data showed an elevated white blood cell count $(13,570 / \mu l)$ (segmented neutrophils $76 \%$, lymphocytes $14 \%$, eosinophils $1.7 \%$ ) and a normochromic normocytic anemia (hemoglobin $10.4 \mathrm{~g} / \mathrm{dl}$ ). Lactate acid dehydrogenase was elevated to 1,201 U/l. C-reactive protein and erythrocyte sedimentation rate were also elevated (108 $\mathrm{mg} / \mathrm{l}$ and $70 \mathrm{~mm} / \mathrm{h}$, respectively). Plasma creatinine was $1.3 \mathrm{mg} / \mathrm{dl}$ and the urea was $74 \mathrm{mg} / \mathrm{dl}$. The 24 -hour urine output was $3,100 \mathrm{ml}$ with a glomerular filtration rate of $65 \mathrm{ml} / \mathrm{min} / 1.73 \mathrm{~m}^{2}$. Urine antigen tests for Legionella pneumophila and Streptococcus pneumoniae were negative. Chest X-ray revealed bilateral diffuse alveolar infiltrates (fig. 1). Respiratory tract infection was suspected and empiric antimicrobial treatment was started with a fluoroquinolone (moxifloxacin). A pharyngeal swab was obtained and a prompt urinalysis showed proteinuria at $600 \mathrm{mg} / 24 \mathrm{~h}$, hematuria with a large number of dysmorphic red blood cells and several red-cell casts. A few hours later, a pulmonary function testing revealed a $\mathrm{FEV}_{1}$ of 2.41 (64.7\% of predicted), FVC of 3.41 (69.0\% of predicted), $\mathrm{FEV}_{1} / \mathrm{FVC}$ ratio of $77.1 \%$, total lung capacity of 5.51 ( $76.3 \%$ of predicted) and diffusing capacity for car-

The presentation of our case report was approved by the Institutional Ethics Committee of 'Attikon' University Hospital, National and Kapodistrian University of Athens, Greece. No additional medical intervention was practiced apart from standard patient care in our institution. Written informed consent was obtained.

\section{KARGER}

Fax +4161306 1234

E-Mail karger@karger.ch

www.karger.com (c) 2010 S. Karger AG, Basel

$0025-7931 / 10 / 0796-0511 \$ 26.00 / 0$

Accessible online at:

www.karger.com/res
Lykurgos Kolilekas, MD

2nd Pulmonary Department, 'Attikon' University Hospital

Athens Medical School, National and Kapodistrian University of Athens

1 Rimini Street, GR-12462 Athens (Greece)

Tel. +30 210583 2361, Fax +30 210532 6414, E-Mail lykol@yahoo.gr 
Fig. 1. Chest X-ray on admission. Diffuse bilateral alveolar infiltrates, predominantly in middle and lower lung regions.

Fig. 2. High-resolution CT of the chest. Extensive homogeneous 'ground-glass' opacities in both lungs, most pronounced in the lower lobes.

bon monoxide (DLCO) of $10.9 \mathrm{mmol} / \mathrm{min} / \mathrm{kPa}(108 \%$ of predicted). A subsequent high-resolution CT of the chest demonstrated diffuse alveolar infiltrates and ground glass opacities (fig. 2). Bronchoscopy was performed thereafter, and hemorrhagic fluid was obtained from bronchoalveolar lavage (BAL), becoming bloodier from the first to the last suctioned syringe (fig. 3). Bronchial washing and BAL cultures were sterile both for common
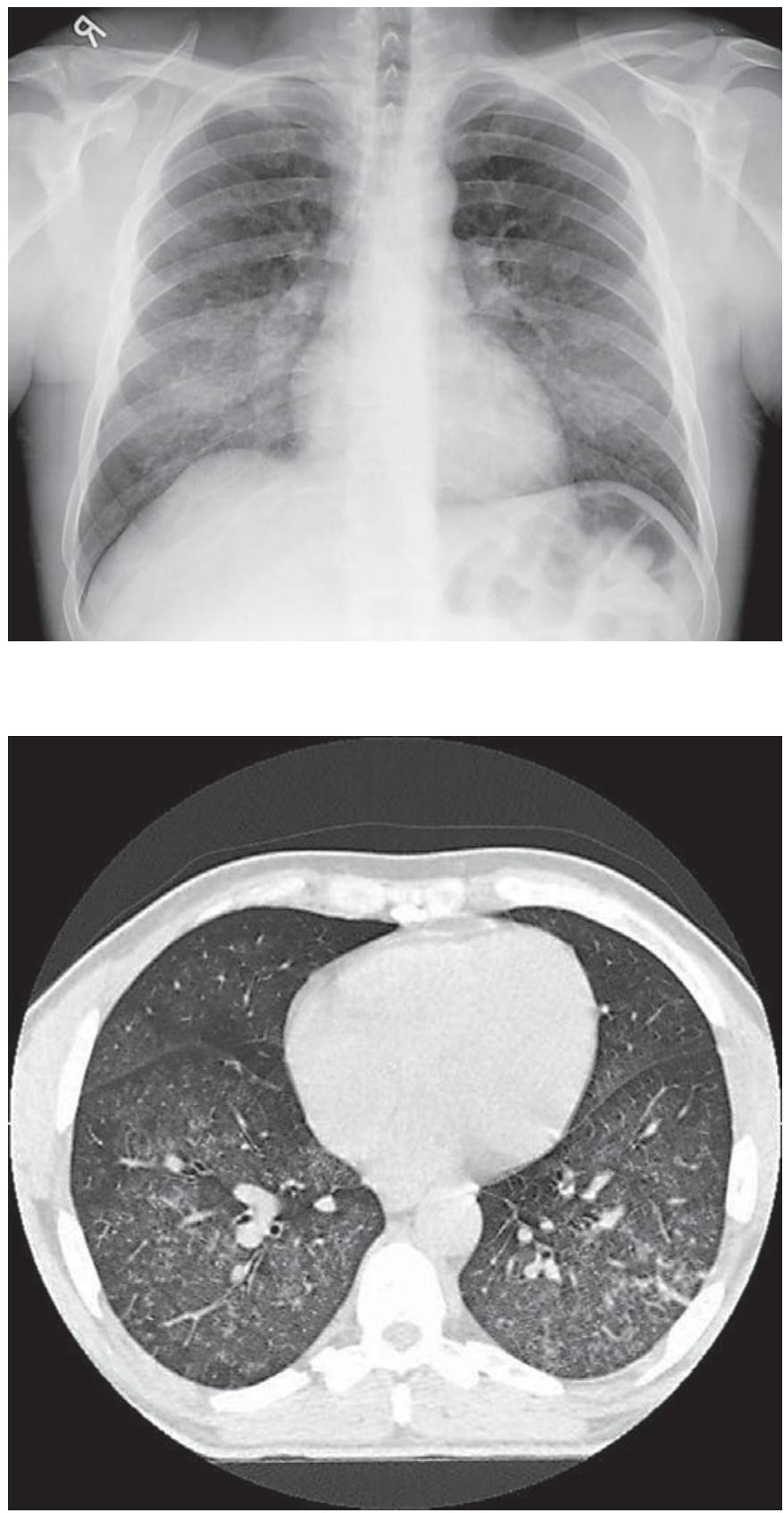

pathogens and acid-fast bacilli. Autoantibodies including cytoplasmic ANCA (c-ANCA), perinuclear ANCA (pANCA), anti-glomerular basement antibodies (antiGBM), antinuclear antibody (ANA), anti-dsDNA antibody, Scl-70, anti-SM, ACA, anti-RNP, cryoglobulins, and anti-phospholipid antibodies were all negative. Serum C3, C5, CH50, IgG, IgM, IgA, and IgE were normal. The anti-streptolysin-O titer (ASTO) was elevated at 526 
Fig. 3. BAL fluid. Bloody lavage fluid, which ranges from pink to red, characteristic of DAH.
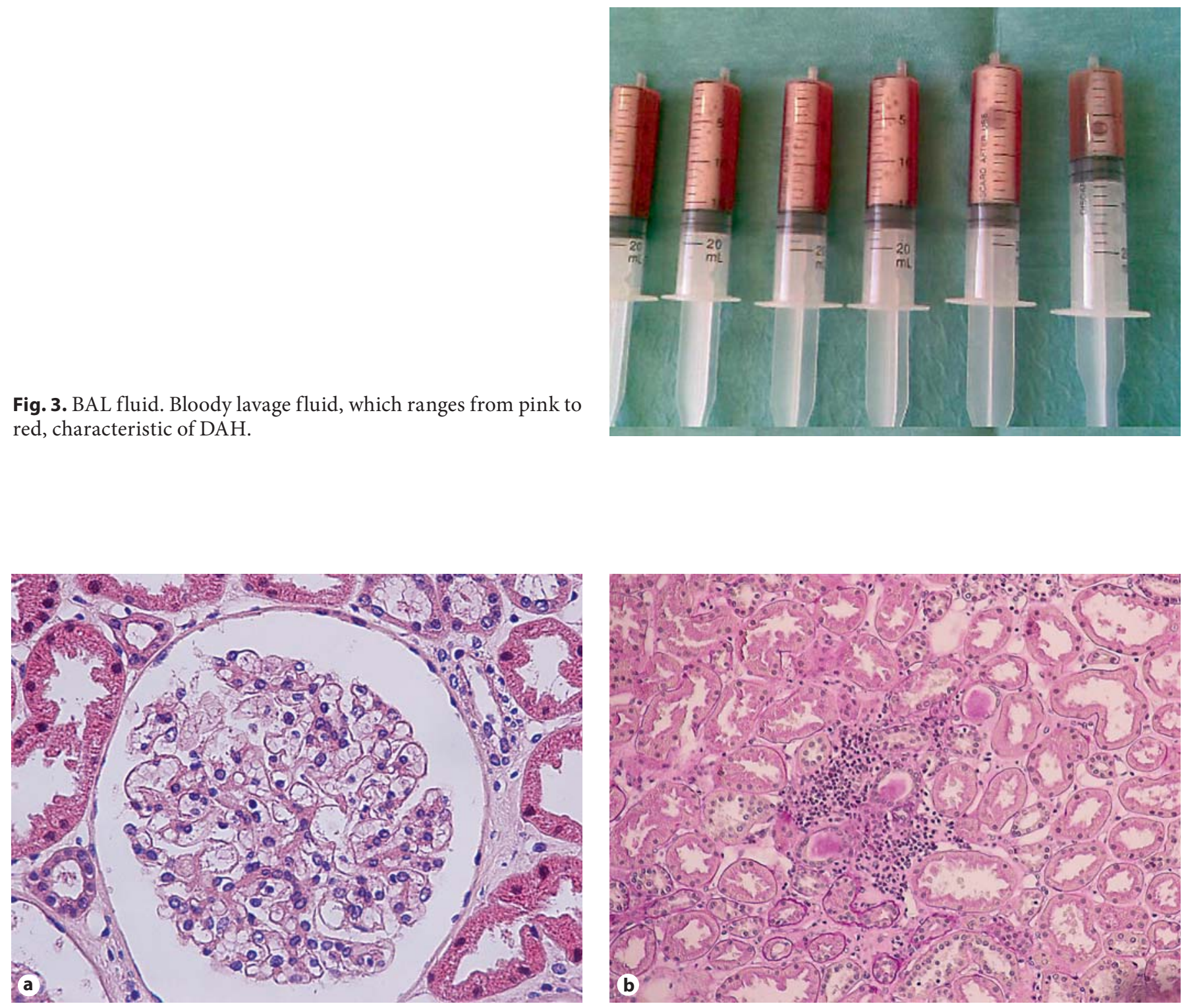

Fig. 4. Kidney biopsy. a Mild lesions of segmental hyperplasia of the mesangial cells and increased mesangial substance. b Interstitial edema with focal infiltration of isolated mononuclear cells. Fibrosis is observed locally. HE. $\times 400$.

(0-240 IU/ml). Serology for HIV, HBV, HCV, CMV, EBV, HSV, L. pneumophila, Chlamydia pneumoniae, Mycoplasma pneumoniae, Coxiella burnetii, Coxsackie, and Leptospira was negative. Blood and urine cultures were negative. A group A $\beta$-hemolytic Streptococcus was cultured from the pharyngeal swab. Based on the findings of the active urine sediment in combination with the diffuse alveolar hemorrhage, a percutaneous kidney biopsy under CT guidance was performed. Glomeruli showed mesangial widening with scattered inflammatory cells and rare tubular atrophy (fig. 4). Immunofluorescence revealed mesangial deposits of $\mathrm{C}^{++}$without deposits of $\operatorname{Ig} \mathrm{A}, \mathrm{IgG}, \mathrm{IgM}, \mathrm{Clq}$ and $\kappa$ or $\lambda$ chains. No crescents were found, nor vasculitis.

What is your diagnosis? 


\section{Diagnosis: Post-Streptococcal Pulmonary-Renal Syndrome}

\section{Discussion}

Pulmonary-renal syndrome is defined as the combination of diffuse alveolar hemorrhage (DAH) and glomerulonephritis [1]. The underlying pulmonary pathology usually consists of small-vessel vasculitis involving arterioles, venules and alveolar capillaries (necrotic pulmonary capillaritis). DAH is the result of the disruption of perfusion and of continuity of pulmonary capillary wall [2].

The catalogue of pulmonary-renal syndromes consists of ANCA-positive and ANCA-negative systemic vasculitis, Goodpasture's syndrome, idiopathic pulmonaryrenal syndrome, drug-associated ANCA-positive vasculitis, autoimmune rheumatic diseases, thrombotic microangiopathy, and DAH complicating idiopathic pauciimmune glomerulonephritis [3].

The underlying renal pathology in the majority of cases of pulmonary-renal syndrome is a form of focal proliferative glomerulonephritis. Fibrinoid necrosis and microvascular thrombi are frequently seen. Extensive crescent formation regularly accompanies glomerular tuft disease. Interstitial infiltration, fibrosis and tubular atrophy are poor prognostic factors. Necrotizing granulomas and small-vessel vasculitis are rare findings. Immunofluorescence helps to distinguish among anti-GBM disease (linear deposition of $\operatorname{IgG}$ ), lupus and post-infectious glomerulonephritis (granular deposition of immunoglobulin and complement), and necrotizing vasculitis (pauci-immune glomerulonephritis) - each one indicating the underlying disease [3].

Based on the clinical picture, laboratory and bronchoscopic findings and the histologic pattern of the kidney biopsy, the most relevant diagnosis, in our case, was acute post-streptococcal glomerulonephritis (PSGN) with DAH.

PSGN is the prototypical post-infectious glomerulonephritis. Glomerulonephritis usually develops 10 days after pharyngitis or 2 weeks after skin infection (impetigo) with a nephritogenic strain of group A hemolytic Streptococcus [4]. Gross hematuria, oliguric acute renal failure, edema, and hypertension are the classic manifestations, however most patients present with a milder disease [5]. It is a very unusual event for DAH to develop in association with PSGN [6-8].

The histopathological patterns of PSGN range from mild proliferative cases to the classical pathology seen in acute PSGN [9]. In our case, the histopathological pattern of the kidney biopsy was consistent with a form of PSGN with mild proliferative lesions and scanty infiltration with inflammatory monocytes. These findings along with the absence of IgG, the normal blood values of C3 and the rapid improvement of the patient with treatment, were suggestive of a PSGN at the resolution phase. In the literature, the acute phase in typical forms of PSGN lasts 1-2 weeks and may resolve in 4-14 days and the complement values although depressed at the acute phase, in $>90 \%$ cases return to normal as the disease subsides [10]. Therefore, the histopathological pattern of our patient, although not specific, was, in association with the laboratory findings, the clinical presentation of renal impairment 2 weeks following a microbiologically documented streptococcal tonsillitis and the rapid and positive response to treatment strongly suggestive of PSGN.

The pathogenic mechanism to explain the presence of DAH with PSGN is not well established. Immune complex deposition on bronchoalveolar epithelium and capillary basement membrane may induce an acute inflammatory reaction with a neutrophil-mediated disruption of the alveolar bed and capillary basement membrane. These events may lead to capillaritis and DAH $[11,12]$.

Therapy relies on aggressive immunosuppression. To this end, the assessment of disease severity such as that proposed by the European Vasculitis Study Group (EUVAS) is recommended [13]. Moreover, therapy is often divided into a two-part model, an initial 'remissioninduction' phase, in which more intensive immunosuppressive therapy is used to control active disease, and a 'maintenance' phase, in which less intensive therapy is used, minimizing the adverse side effects while still maintaining disease remission [11].

In our case, the patient was treated initially with intravenous bolus administration of methylprednisolone $(1 \mathrm{~g} /$ day for 3 days) and a single intravenous dose of cyclophosphamide $(750 \mathrm{mg})$. Treatment continued with prednisolone in doses of $25 \mathrm{mg} /$ day orally. After 6 days of treatment, chest X-ray showed significant amelioration with withdrawal of the alveolar infiltrates, oxygenation significantly improved $\left(\mathrm{S}_{\mathrm{a}} \mathrm{O}_{2}=98 \%\right)$ and urinalysis returned to normal. Pulmonary function studies showed an improvement in the restrictive pattern $\left(\mathrm{FEV}_{1}: 3.5 \mathrm{l}\right.$, $86.3 \%$ of predicted, FVC: $4.41,89.3 \%$ of predicted with a $\mathrm{FEV}_{1} / \mathrm{FVC}$ ratio of $79.5 \%$ ) and a normalization of DLCO $(8.0 \mathrm{mmol} / \mathrm{min} / \mathrm{kPa}, 70.8 \%$ of predicted). Twelve days af- 
ter admission the patient had normalized ASTO levels and was discharged without symptoms.

In conclusion, the abrupt presentation of diffuse alveolar infiltrates, in accordance with novel findings of renal involvement post-streptococcal infection of the oropharynx, should alert the clinician for the development of pulmonary-renal syndrome, which necessitates simultaneously prompt diagnostic and therapeutic interventions - a run-and-gun affair.

\section{Key Words}

Pulmonary hemorrhage $\cdot$ Renal diseases $\cdot$ Bronchoalveolar lavage

\section{References}

1 Gallagher H, Kwan J, Jayne RW: Pulmonary renal syndrome: a 4-year, single-center experience. Am J Kidney Dis 2002;38:42-47.

2 Davies DJ: Small vessel vasculitis. Cardiovasc Pathol 2005; 14:335-346.

3 Papiris SA, Manali ED, Kalomenidis I, et al: Bench-to-bedside review: pulmonary-renal syndromes - an update for the intensivist. Critical Care 2007;11:213.

4 Hahn RG, Knox LM, Forman TA: Evaluation of poststreptococcal illness. Am Fam Physician 2005;71:1949-1954.

5 Sarkissian A, Papazian M, Azatian G, et al: An epidemic of acute postinfectious glomerulonephritis in Armenia. Arch Dis Child 1997;77:342-344.
6 Gilboa N, McIntire S, Hopp L, et al: Acute noncrescentic poststreptococcal glomerulonephritis presenting with pulmonary hemorrhage. Pediatr Nephrol 1993;7:147-150.

$\checkmark 7$ Chugh KS, Gupta VK, Singhal PC, et al: Case report: poststreptococcal crescentic glomerulonephritis and pulmonary hemorrhage simulating Goodpasture's syndrome. Ann Allergy 1981;47:104-106.

$\checkmark 8$ Sung HY, Lim CH, Shin MJ, et al: A case of post-streptococcal glomerulonephritis with diffuse alveolar hemorrhage. J Korean Med Sci 2007;22:1074-1078.

9 Yoshizawa N, Suzuki Y, Oshima S, et al: Asymptomatic acute poststreptococcal glomerulonephritis following upper respiratory tract infections caused by group A streptococci. Clin Nephrol 1996;46:296-301.
10 Benudiz N: Recognizing the elusive signs and symptoms of PSGN. JAAPA 2007;20:2025.

11 Fukuda Y, Yamanaka N, et al: Immune complex-mediated glomerulonephritis and interstitial pneumonia simulating Goodpasture's syndrome. Acta Pathol Jpn 1982;32: 361-370.

12 Green RJ, Ruoss SJ, Kraft SA, et al: Pulmonary capillitis and alveolar hemorrhage. Update on diagnosis and management. Chest 1996;110:1305-1316.

13 Frankel SK, Cosgrove GP, Fischer A, et al: Update in the diagnosis and management of pulmonary vasculitis. Chest 2006;129; 452 465. 